\title{
Insights into Twinning in Mg AZ31: A Combined EBSD and Machine Learning Study
}

\author{
${ }^{1}$ Andrew Orme, ${ }^{1}$ Isaac Chelladurai, ${ }^{1}$ Travis M. Rampton, ${ }^{1}$ David T. Fullwood, ${ }^{3}$ Ali Khosravani \\ ${ }^{2}$ Michael P. Miles, ${ }^{4}$ Raja K. Mishra \\ ${ }^{1}$ Mechanical Engineering department, Brigham Young University, Provo, UT \\ ${ }^{2}$ Manufacturing Technology department, Brigham Young University, Provo, UT \\ ${ }^{3}$ Woodruff School of Mechanical Engineering, Georgia Institute of Technology, Atlanta, GA \\ ${ }^{4}$ General Motors R\&D Center, Warren, MI
}

\section{Keywords \\ Magnesium AZ31 \\ Twin Formation \\ Machine Learning \\ Decision Tree \\ EBSD}

\begin{abstract}
To explore the driving forces behind deformation twinning in $\mathrm{Mg}$ AZ31, a machine learning framework is utilized to mine data obtained from electron backscatter diffraction (EBSD) scans in order to extract correlations in physical characteristics that cause twinning. The results are intended to inform physics-based models of twin nucleation and growth. A decision tree learning environment is selected to capture the relationships between microstructure and twin formation; this type of model effectively highlights the more influential characteristics of the local microstructure. Trees are assembled to analyze both twin nucleation in a given grain, and twin propagation across grain boundaries. Each model reveals a unique combination of crystallographic attributes that affect twinning in the Mg. Twin nucleation is found to be mostly controlled by a combination of grain size, basal Schmid factor, and bulk dislocation density while twin propagation is affected most by grain boundary length, basal Schmid factor, angle from grain boundary plane to the RD plane, and grain boundary misorientation. The machine
\end{abstract}


learning framework can be readily adapted to investigate other relationships between microstructure and material response.

\section{BACKGROUND}

With the increasing demand for materials with high strength-to-weight ratios in transportation applications, magnesium alloys have emerged as competitive alternatives for structural components [1]. However, low formability at room temperatures prevents $\mathrm{Mg}$ from being a cost effective solution in mass production applications [2-10]. One issue that leads to this unfavorable forming characteristic is the strong basal texture of rolled $\mathrm{Mg}$ sheets, which forms during the rolling process. The hexagonal close packed (HCP) crystal structure of $\mathrm{Mg}$, combined with the rolled texture of the sheet material, limits the availability of readily activated slip systems. The absence of easy slip systems to accommodate the applied strain leads to deformation twinning [3, 6, 8, 11-15]. While they can help accommodate strain, twins can also serve as nucleation sites for cracks $[6,11,15]$. The current work aims to elevate understanding of relations existing between microstructure characteristics and twin formation at room temperature in $\mathrm{Mg}$ alloys.

The nucleation of a deformation twin in $\mathrm{Mg}$ depends upon so many variables that it may be considered a stochastic event [16-21]. In order to begin to unravel the complexity behind the physics of twin deformation, many observations of nucleation events are required (as manifested by several of the studies quoted above). Once sufficient data is available, statistical analysis can be applied in various forms to extract correlations linking the observed structure and applied field variables to nucleation and propagation events.

Electron backscatter diffraction (EBSD) offers an ideal data collection technique to observe nucleation events, with the ability to scan hundreds of sample points per second [22-24]. Furthermore, recently developed high-resolution (HR-EBSD) techniques (also referred to as cross-correlation EBSD) provide new levels of microstructure data that include improved quantification of lattice orientation, local relative strains and dislocation density that may critically affect twin formation [25-28]. 
Machine learning is a suitable statistical analysis approach for mining large quantities of data, such as that from HR-EBSD methods. Already established in various fields as a reliable method for extracting insights and knowledge of relations between attributes in vast databases, machine learning provides a framework whereby complex events, such as deformation twinning, can be connected with local structure and field parameters [29-32]. In this paper, a J48 machine learning algorithm will be employed to create predictive models for twin events in grains, as observed by EBSD, in the form of decision trees. A decision tree framework is particularly valuable for its interpretability. Using this approach, instances of nucleated and propagated twins in Mg alloy AZ31 will be correlated with microstructure attributes and the relative importance of these attributes in triggering the physical phenomena will be investigated.

Using this proposed machine learning framework, the paper aims to achieve the following:

1. Create decision tree models for describing twin nucleation in grains and twin propagation across grain boundaries in the $\mathrm{Mg}$ alloy $\mathrm{AZ31}$ by extracting attribute based rules from EBSD data.

2. Rank the physical factors according to influence on the nucleation or propagation of twins.

3. Demonstrate that the proposed approach provides a reliable machine learning framework for mining EBSD data, such that future studies of rare and complex events may be accelerated.

\subsection{Deformation Twinning in Magnesium}

Slip in Mg depends upon a complex array of potential slip systems [14, 33-35]: basal <a> ( 2 independent), prismatic <a> $(2$ independent $)$, pyramidal < a > type I ( 4 independent $)$, and pyramidal $<\mathrm{c}+\mathrm{a}>$ ( 5 independent). Furthermore, plastic deformation can be accommodated by compression twinning (predominantly the six $\{10 \overline{1} 1\}\{10 \overline{1} \overline{2}\rangle$ variants) and tensile twinning (mainly the six $\{10 \overline{1} 2\}\langle 10 \overline{1} \overline{1}\rangle$ variants [36]). The Taylor model of plastic deformation requires that at least 5 independent slip systems be activated to accommodate an arbitrarily imposed strain [37]. While there is a sufficient number of slip systems in $\mathrm{Mg}$ to accommodate the Taylor model, only the basal system and prismatic $<a>$ systems are easily activated at room temperature, providing only 4 independent active slip systems (see Table 1). This leaves a requirement of one additional 
deformation mechanism for compliance with the Taylor criterion. Twin activity can provide the necessary 5 th degree of freedom for deformation.

The interplay between slip and twin activity can most simply be described by a CRSS model. In the case of rolled AZ31 sheet, the strong basal texture requires some slip or twinning along the $\langle\mathrm{c}\rangle$ or $\langle\mathrm{c}+\mathrm{a}\rangle$ directions in order to accommodate contraction or extension of the c-axis [20]. The much higher $\tau$ crss values of the $<c+a>$ slip systems relative to those for tensile twins (see Table 1 for ranges of values reported in the literature) indicates that tensile twinning will occur before $\langle\mathrm{c}+\mathrm{a}>$ slip to provide the 5 th active system in the Taylor model [3, 14, 33, 38-40]. However, such an approach is not adequate to describe the complex response that is seen in practice.

Table 1: Ranges of published $\tau_{\text {crss }}$ values for deformation mechanisms in $\mathrm{Mg}$ at room temperature.

\begin{tabular}{|l|c|c|l|c|c|}
\hline Slip System & basal <a> & prismatic $<\mathrm{a}>$ & $\begin{array}{l}\text { pyramidal } \\
<\mathrm{c}+\mathrm{a}>\end{array}$ & $\{10 \overline{1} 2\}$ twinning & $\{10 \overline{1} 1\}$ twinning \\
\hline $\boldsymbol{\tau}_{\text {crss }}(\mathbf{M P a})$ & 4 & $8-10$ & $80-100$ & $11-12$ & $76-153$ \\
\hline
\end{tabular}

For example, work by Barnett et al showed that twin nucleation in Mg follows a HallPetch relationship, where the required twinning stress increases with smaller grain size [16]. Furthermore, Beyerlein et al recently combined atomistic simulations with an extensive EBSDbased study to demonstrate the effects of grain boundary (GB) misorientation and GB dislocation structure on nucleation of twins [17]. The resultant model utilized a stochastic approach to twin nucleation and combined it with a CRSS based model for twin propagation. It proposed distinct weights for the probability of twin nucleation on grain boundaries above and below $45^{\circ}$ misorientation, due to the observed tendency of twins to be present at low angle GBs. A similar study by Khosravani [41] further categorized twin nucleation events at GBs into spontaneous formation of twins (slip-assisted nucleation) and propagation of twins across grain boundaries (twin-assisted nucleation). This paper also highlighted the importance of dislocation structure near GBs. The study further demonstrated that twins easily propagate through low angle $\left(15-25^{\circ}\right)$ GBs and tend to nucleate at high angle GBs $\left(>39^{\circ}\right)$. The different considerations of these various studies and models might be reconciled into one framework via a different approach, in which large data sets are explored using machine learning to reveal correlations to form the basis for model structure and parameters. 


\subsection{Electron Backscatter Diffraction (EBSD)}

The automated acquisition of EBSD data has been used in materials science for several decades, culminating in tools that achieve common data collection speeds of hundreds of points per second. Such speeds allow for relatively large microstructures to be quickly and accurately measured $\left(\sim 0.3^{\circ}\right.$ resolution in lattice orientation [42]). The gathered EBSD data can then be processed by commercially available software (e.g. OIM ${ }^{\mathrm{TM}}$ ) to produce other crystallographically significant data related to grain orientation, phase, and morphology [43]. Available information from traditional EBSD techniques also includes grain size distribution, Schmid factors, variations (gradients) in lattice orientation, and GB misorientations.

Additionally, in recent years high resolution EBSD (HR-EBSD) techniques have been developed to extract even more information from the collected EBSD data [25-28]. These methods apply cross-correlation techniques to EBSD images in order to measure orientations and relative crystal rotations with even greater angular resolution $\left(0.006^{\circ}\right)$. Using the crosscorrelation technique, HR-EBSD is also capable of being used to measure (relative) elastic strain and geometrically necessary dislocation (GND) density at each data point. Both measurements rely on the accurate extraction of the elastic distortion gradient, providing reliable strain and GND fields over large scan areas [44-47].

With the combination of HR-EBSD data and standard EBSD metrics the mechanisms underlying deformation twinning of $\mathrm{Mg}$ can be more fully characterized. Microstructure characteristics analyzed in this paper will include grain size, kernel average misorientation, geometrically necessary dislocations, orientation of the c-axis relative to the sheet normal direction, grain boundary misorientations, and the Schmid factors for $\langle a>$ type slip, $<c+a>$ type slip, and twinning. These attributes provide a broad set of crystallographic measurements that may relate to the nucleation and propagation of twinning in AZ31.

\subsection{Machine Learning}

With the abundance of crystallographic information that may affect twinning in AZ31, and given the uncertainty underlying the cause and effect of twin nucleation and propagation, a modeling approach that minimizes assumptions made about the nature of the events under investigation may provide previously unidentified insights into the actual causes of these events. Machine learning, inclusive of various types of data mining developed to find statistical 
correlations among large datasets, offers one such method of non-discriminatory characterization [29-32]. The basic idea of machine learning is to create relationships between user defined attributes (such as microstructure characteristics) and an outcome (such as a twinning event), referred to as a class. This is performed by training an algorithm to predict the resulting class, or outcome, based on a set of training instances containing attribute and class data. The trained algorithm can then be tested against other sets of instances for accuracy, with potential to be deployed as a predictive model if accuracy and precision meet user specifications. By employing this approach to predict twin activity in AZ31, the resulting machine learning models will help confirm whether all important aspects have been incorporated into current statistical $\mathrm{Mg}$ twinning models and may give insights into what attributes are missing.

Machine learning has been utilized in various areas of materials science and engineering to develop constitutive relations that establish structure property relationships [48-54]. Many such studies treat the resultant machine learning models as a purely black box approach, used to predict an event without consideration to interpreting the decision framework behind the prediction. This is particularly true for certain algorithms employed by these studies, such as neural networks or naïve bayes predictors, which while providing high levels of accuracy, do not have easily interpretable decision hierarchies. In contrast, the primary goal of this paper is to create, via machine learning, comprehensible models which describe twinning phenomena in $\mathrm{Mg}$ and then use those models to elucidate the physics associated with these events.

Machine learning algorithms can be broken down into three categories: knowledgebased, rule-based, and skill-based [55]. Knowledge-based learning is equivalent to ab-initio studies and therefore requires a greater prior understanding of the studied phenomenon. Rulebased models however, provide less structured connections while still maintaining a moderate level of accuracy. Finally, skill-based algorithms can be compared to complex curve fitting in which the resultant model provides an easily implementable mathematical equation but may have reduced physical significance and interpretability. For the case of twinning in AZ31, a rule-based method is most suitable, given that there is not enough information to develop an accurate knowledge-based model, and skill-based models would not provide physically interpretable insights into the causes of twinning.

The desire for an easily interpretable rule based classifier led to the selection of the J48 decision tree classifier. The J48 classifier expands the functionality of the $\mathrm{C} 4.5$ algorithm by 
allowing for classification of continuous variables [56]. J48 produces easily comprehensible decision trees. During training, J48 categorically partitions data to maximize the information gain at each level of the tree structure, where information gain is a mathematically defined property. The result is a hierarchy of attribute (e.g. microstructure property) based divisions that results in the selection of a particular class, or an outcome (such as twin formation), for a given instance. Figure 1 is an example of a decision tree created using the $\mathrm{J} 48$ classifier. The tree hierarchy is composed of a root, multiple branches, and multiple leaves, respectively labeled as "R," "B," and "L". The predicted class for each instance is then tested against the actual class for that instance and the accuracy of the tree is evaluated.

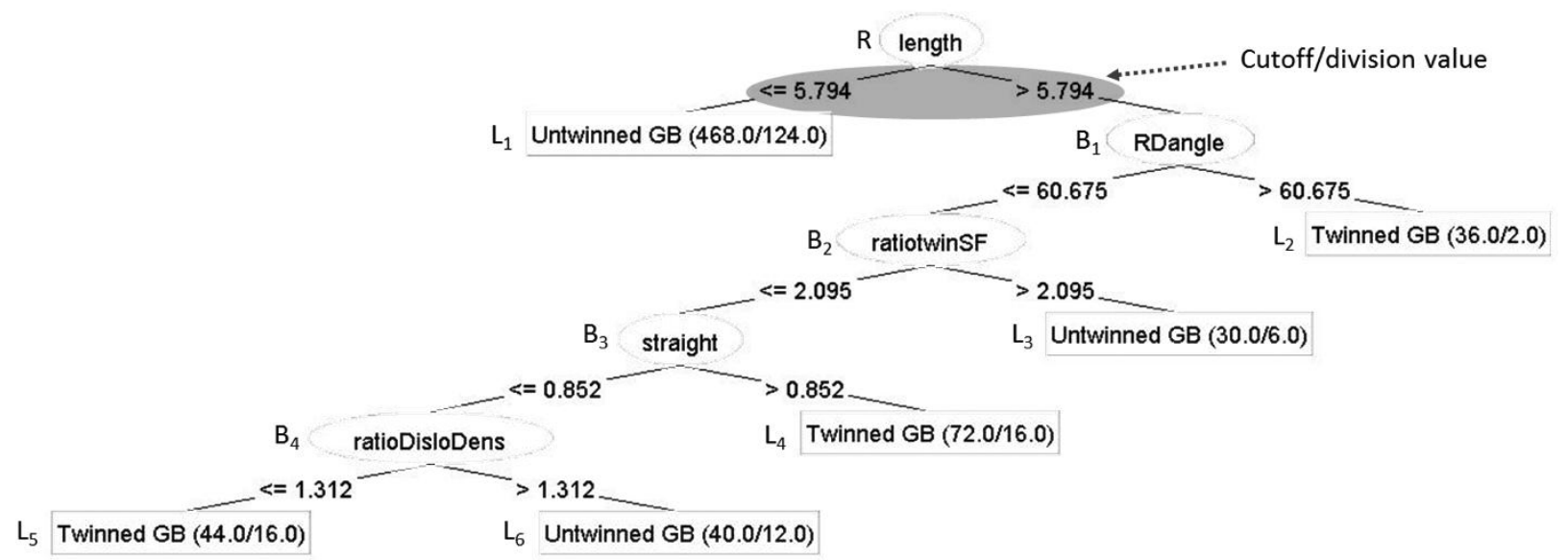

Figure 1: A typical decision tree and the associated hierarchy (R: Root, B: Branch, and L: Leaf) present in a decision tree. Note that the values inside the leaves appear in the following format: (\# of classified instances / incorrect predictions). The relationships in this tree are provided for illustration and are not part of the investigation at hand.

By definition, attributes found nearer to the beginning of the tree, or "root", provide greater information gain than subsequent attributes and are thus considered to be more influential in determining the outcome. "Branches" represent subsequent divisions and "leaves" represent a predicted class outcome. Hence smaller decision trees are desirable since they only retain attributes that have the greatest impact on a particular phenomenon. With only the most pertinent features captured, the resultant model becomes more physically understandable.

Thus the model created by a decision tree may be interpreted or applied in several ways:

i) as a model/constitutive relation for the studied event.

ii) as a set of insights into the causes of the event that help focus further research. 
iii) as a way to determine/capture more events for further study by predicting when / where critical events will occur.

The tree also provides a framework to systematically increase the data set from which to train the algorithm and thus refine the resulting model. In the case of time-consuming data collection procedures, the potential for a refined data collection method is desirable as it has the ability to intelligently guide data collection towards areas of interest, as defined by the information contained in the decision tree. Such an approach to refined data collection is an area of ongoing research and beyond the scope of this paper.

\section{MATERIALS AND METHODS}

The material studied in this research was a $3 \mathrm{~mm}$ thick cold rolled and annealed AZ31 $\mathrm{Mg}$ alloy plate with an initially twin-free microstructure. Specimens were prepared as $3 \mathrm{~mm} \mathrm{x}$ $4 \mathrm{~mm} \times 3 \mathrm{~mm}$ square prisms, cut from a fully annealed AZ31 plates, using wire EDM to minimize the amount of deformation on the sample surface. The samples were then prepared for EBSD study by mechanical polishing using suitably sized diamond abrasives followed by an OP-S colloidal silica slurry polish. Finally, the sample was etched with a solution of $60 \%$ ethanol, $20 \%$ distilled water, $15 \%$ acetic acid and 5\% nitric acid. Using a focused ion beam, platinum fiducial marks were deposited on the specimen surface to measure approximate 2-dimensional strain in the scan area [41].

The first specimen was compressed along the rolling direction (RD) up to $\sim 3 \%$ strain, and the second specimen was strained slightly further to $\sim 3.5 \%$. This strain was chosen because it was the point at which there was sufficient twin nucleation and propagation for detailed analysis using machine learning, based on prior work [57].

EBSD scans were carried out on an FEI-Helios NanoLab ${ }^{\mathrm{TM}}$ 600i SEM equipped with $\mathrm{OIM}^{\mathrm{TM}}$ data acquisition software and a high-speed Hikari ${ }^{\mathrm{TM}}$ camera [43, 58]. A step size of 300 $\mathrm{nm}$ was selected for two scan areas of $84 \times 84 \mu \mathrm{m}^{2}$ on the first sample and $70 \times 270 \mu \mathrm{m}^{2}$ on the second. Subsequent post processing of data utilized $\mathrm{OIM}^{\mathrm{TM}}$ Analysis, Matlab ${ }^{\mathrm{TM}}$, and OpenXY (open source HR-EBSD software [59]) to organize attributes for input into the machine learning environment $[58,60,61]$. The extracted attributes, their origin, and a brief explanation are listed in Table 2. 
The analysis presented in this paper calls for two separate models to be created, one for nucleation of twins in grains and another for propagation of twins across grain boundaries. For this study, a nucleation event was classified by identifying at least one twin in a grain (i.e. both twins that spontaneously formed in a grain and twins that propagated into the grain from a neighboring grain). A propagation event was defined when at least one twin was present on either side of a given GB. The nucleation model took into consideration attributes within a grain, but ignored GB morphology. The propagation model, on the other hand, focused on parameters relating to GBs.

Several attributes were selected to represent morphological data (e.g. grain size, number of neighbors, neighboring grain size, GB length). Attributes relating to feature size play a large role in both current Hall-Petch type models $[12,16]$ and stochastic models $[17,62]$.

Other attributes (such as GB trace orientation, GB straightness, grain orientation relative to loading direction, Schmid factors, kernel average misorientation and the related metric of GND density) may affect strain compatibility, incentive to slip or twin, and information regarding localization of deformation. Kernel average misorientation (KAM) and GND content, measureable by HR-EBSD, indicate strain localization in a material, potentially triggering twin activity. The Schmid factor of a particular slip or twin system also serves as an indicator of the likelihood of the relevant activity.

Table 2: Input attributes (parameters) for machine learning. Check marks indicate attributes utilized as inputs for creating each model (nucleation or propagation). Highlighted check marks indicate the important microstructural features as found by each machine learning model. (N: nucleation, $P$ : propagation).

\begin{tabular}{|c|c|c|c|c|}
\hline \multirow{2}{*}{$\begin{array}{l}\text { Attribute (Abbreviation in } \\
\text { decision tree) }\end{array}$} & \multirow[t]{2}{*}{ Description } & \multirow[t]{2}{*}{ Source } & \multicolumn{2}{|c|}{ Model } \\
\hline & & & $\mathbf{N}$ & $\mathbf{P}$ \\
\hline Grain size (SIZE) & $\begin{array}{l}\text { Equivalent diameter. Calculated as the diameter } \\
\text { of a circle with the same area as the measured grain }\end{array}$ & $\begin{array}{l}\text { OIM } \\
\text { Analysis }\end{array}$ & $\checkmark$ & \\
\hline Neighboring grain size (NBRSIZE) & - Average neighbor grain size & & $\checkmark$ & \\
\hline Relative grain size (RELSIZE) & - Grain size divided by neighboring grain size & & $\checkmark$ & \\
\hline Number of neighbors (NUMNBRS) & - number of neighboring grains & & $\checkmark$ & \\
\hline $\begin{array}{l}\text { Deviation of c-axis from RD, TD, } \\
\text { and ND }(R D M I S O, N D M I S O \text {, } \\
T D M I S O) *\end{array}$ & - Smallest angle of misorientation & & $\checkmark$ & $\checkmark$ \\
\hline $\begin{array}{l}\text { Kernel average misorientation } \\
(\mathrm{MISO}) *\end{array}$ & $\begin{array}{l}\text { - Average misorientation of directly neighboring } \\
\text { points (with } 5^{\circ} \text { cutoff) }\end{array}$ & & $\checkmark$ & $\checkmark$ \\
\hline $\begin{array}{l}\text { Schmid factors }(\mathrm{SF}) \text { of basal }<\mathrm{a}> \\
\text { and pyramidal }<\mathrm{c}+\mathrm{a}>\text { slip systems } \\
(B A S A L S F, C A S F)^{*}\end{array}$ & $\begin{array}{l}\text { - Maximum value of each slip system taken as a } \\
\text { grain average }\end{array}$ & & $\checkmark$ & $\checkmark$ \\
\hline
\end{tabular}




\begin{tabular}{|c|c|c|c|c|}
\hline $\begin{array}{l}\text { Schmid factors of }\{10 \overline{1} 2\}\langle 10 \overline{1} \overline{1}\rangle \\
\text { tensile twins (TWINSF) }\end{array}$ & $\begin{array}{l}\text { - Maximum value, taken as a grain average (also } \\
\text { considers the possibility of negative values) }\end{array}$ & Matlab & $\checkmark$ & \\
\hline $\begin{array}{l}\text { Ratio of twinning Schmid factor to } \\
<\mathrm{c}+\mathrm{a}>\text { Schmid factor }(\text { TWINCASF })^{*}\end{array}$ & $\begin{array}{l}\text { - Maximum SF for twinning divided by max SF for } \\
\text { pyramidal }<c+a>\end{array}$ & & $\checkmark$ & $\checkmark$ \\
\hline GB misorientation (GBMISO) & - Rodriguez misorientation & & & $\checkmark$ \\
\hline GB length $(L E N G T H)$ & - Length along measured GB segment & & & $\sqrt{ }$ \\
\hline GB straightness (STRAIGHT) & $\begin{array}{l}\text { - Average distance of the measured boundary from } \\
\text { a straight line connecting the boundary endpoints }\end{array}$ & & & $\checkmark$ \\
\hline $\begin{array}{l}\text { Approximate GB orientation } \\
(R D A N G L E)^{*}\end{array}$ & - Angle between the GB trace and RD & & & $\checkmark$ \\
\hline $\begin{array}{l}\text { Local dislocation densities } \\
(L O G D D)^{*}\end{array}$ & - Grain average of sum of Nye tensor terms & "HR-EBSD & $\bar{\checkmark}$ & $\checkmark$ \\
\hline
\end{tabular}

*Value for attribute recorded on both sides of a given grain boundary in addition to the average difference in values over the boundary - leading to multiple attributes relating to each of these items. They are labeled in decision trees with the prefix MAX, MIN, or DIFF preceding the attribute label given in Table 2.

The remaining attribute of GB misorientation not only reflects morphology (local relative orientations), but also partially reflects energy associated with GBs. This also relates to GB defects, and has already been linked to twin nucleation and propagation [41, 63].

The metrics collected were gathered for grains that did not twin and grains that twinned, also referred to as parent grains. Grains and grain boundaries near the edge of the scanned area were excluded from consideration due to a lack of complete information for various local attributes (such as grain size).

Once the previously described attributes were collected for each grain and grain boundary, they were organized into a data structures suitable for input into a machine learning program. This study used a publicly available machine learning package, WEKA, for the data mining process [64]. A J48 decision tree classifier was then chosen to find correlations between input parameters and twinning [65]. The algorithm employs a method that increases the information gain of the data as it is partitioned at each node of the tree. Information gain for J48 is calculated by the following relations:

$$
\begin{aligned}
& G(S, A)=E(S)-\sum_{v \in \operatorname{Values}(A)} \frac{\left|S_{v}\right|}{|S|} E\left(S_{v}\right) \\
& E(S)=\sum_{i=1}^{c}-p_{i} \log _{2} p_{i}
\end{aligned}
$$

In these equations $\mathrm{S}$ is the total set of instances being considered, $\mathrm{S}_{v}$ is the subset of $\mathrm{S}$ with value $v, \mathrm{~A}$ is the attribute under consideration, $v$ is a particular value of the considered attribute A, c is the set of possible outputs (these being 'twin' or 'no twin' for this study) for 
value $v$, and $\mathrm{p}$ is the probability of instance $i$ having value $v$. Eqn. $l$ calculates the information gained with selection of a particular attribute and Eqn. 2 calculates the entropy of moving toward that attribute. When the information gain for an attribute is higher than a user defined threshold and the entropy required for moving towards this attribute is lower than the entropy required for moving towards other attributes, the algorithm selects this attribute as most suited for forming a branch or root in the decision tree.

In this study the data used to train the algorithm indicates, using a class variable, whether a grain nucleates a twin or not for the first study (nucleation), and whether a grain boundary propagates or blocks a twin for the second study (propagation). Relevant roots, branches and leaves of each decision tree are labeled "R," "B," and, "L" respectively in Figures 2 and 4 in this paper. 
Additional constraints were placed on the machine learning algorithm to avoid arriving at an overly complicated model; these include applying a confidence factor of 0.25 (an algorithm parameter) and disallowing any leaf with less than two instances. The confidence factor value sets a limit on the minimum acceptable information gain for a branch to be included in the tree. Its values are greater than zero, with larger values requiring less information gain per branch. Requiring a minimum of two instances to create a leaf prevents overfitting the model to a particular dataset by reducing the effect of noise or outliers.

The two decision trees (for nucleation and propagation) were initially trained and validated using data from a single scan. They were subsequently tested on a second larger scan. In order to create a valid model using the initial data set, standard 10 fold cross-validation techniques were applied to the decision tree algorithm. Cross-validation is a proven machine learning technique where the data is split into 10 stratified subsets called folds. One fold is removed from the dataset, and the other 9 are used to train the classifier. The removed fold is then used to test the model created by the other 9 . This is repeated with each of the 10 folds used to test against the model formed by the other 9 and a final average model is generalized from the results. A minimum of two instances per leaf was also required as an additional precaution to avoid the effects of over fitting $[30,31]$. Over fitting is an occurrence in machine learning that can cause a model to have poor generalization capabilities (i.e. the model only accurately describes the relatively small subset of training instances).

The models generated for this study used detailed inputs from 104 grains to build the nucleation model and 130 grain boundaries to create the propagation model, from the first specimen described above. Among the 104 grains used in the creation of the nucleation model, 38 had detectable twins present. The nucleation model took into account a total of 13 attributes, taken from Table 2, including grain size, number of neighboring grains, average size of neighboring grains, relative size of a grain compared to its neighbors, kernel average misorientation, deviation of the c-axis from the RD, TD, and ND, basal Schmid factor, pyramidal $<\mathrm{c}+\mathrm{a}>$ Schmid factor, tensile twin Schmid factor, the ratio of the twin Schmid factor to the pyramidal $<\mathrm{c}+\mathrm{a}>$ Schmid factor, and $\log _{10}$ of dislocation density measured by HR-EBSD.

Of the 130 observed grain boundaries that came in contact with one or more twins, 30 were found to facilitate twin propagation. The propagation model took into account a total of 19 attributes, based on attributes listed in Table 2. Included were kernel average misorientation, 
basal Schmid factor, the ratio of the twin Schmid factor to the pyramidal $<c+a>$ Schmid factor, $\log _{10}$ of dislocation density measured by HR-EBSD, and deviation of the c-axis from the ND. These 5 attributes were calculated on both sides of a given grain boundary along with the difference between them. GB straightness, approximate GB orientation, GB length, and GB misorientation were also included.

The second dataset contains 1239 grains and 1127 GBs. This data was used to test the models created with the smaller dataset, giving insight into the accuracy of the models when applied to a more generic dataset.

The overall accuracy of each model was assessed as the total number of correct predictions divided by the total number of instances used to create the model. Each model has two accuracy percentages, the first is the accuracy of the model against the training data, or the smaller dataset, and the second is the accuracy of the model when tested against the larger dataset, which was not used in training. The second accuracy suggests the feasibility of the model in being deployed for use against other independent datasets. Maps of correct and incorrect predictions were also created to visually represent model accuracy in the microstructure. Attributes found to be influential were compared with literature on twinning in $\mathrm{Mg}$ to either confirm previous findings or suggest new areas of investigation. The number of correct predictions versus incorrect predictions at each leaf was also evaluated.

\section{RESULTS}

\subsection{Twin Nucleation in Grains - Results}

The model created for nucleation of twins as a function of microstructure attributes is shown inFigure 2: Decision tree for characterizing twin nucleation within an individual grain. Labels R, $\mathrm{B}$ and $\mathrm{L}$ index the root, branches and leaves, respectively. Attribute labels used in the tree are defined in Table 2.Figure 2. In testing the observed microstructure against the created decision tree, $86.5 \%$ of the 104 grains and $75.1 \%$ of the 1239 grains were correctly categorized by the 
model as either twinning or not twinning.

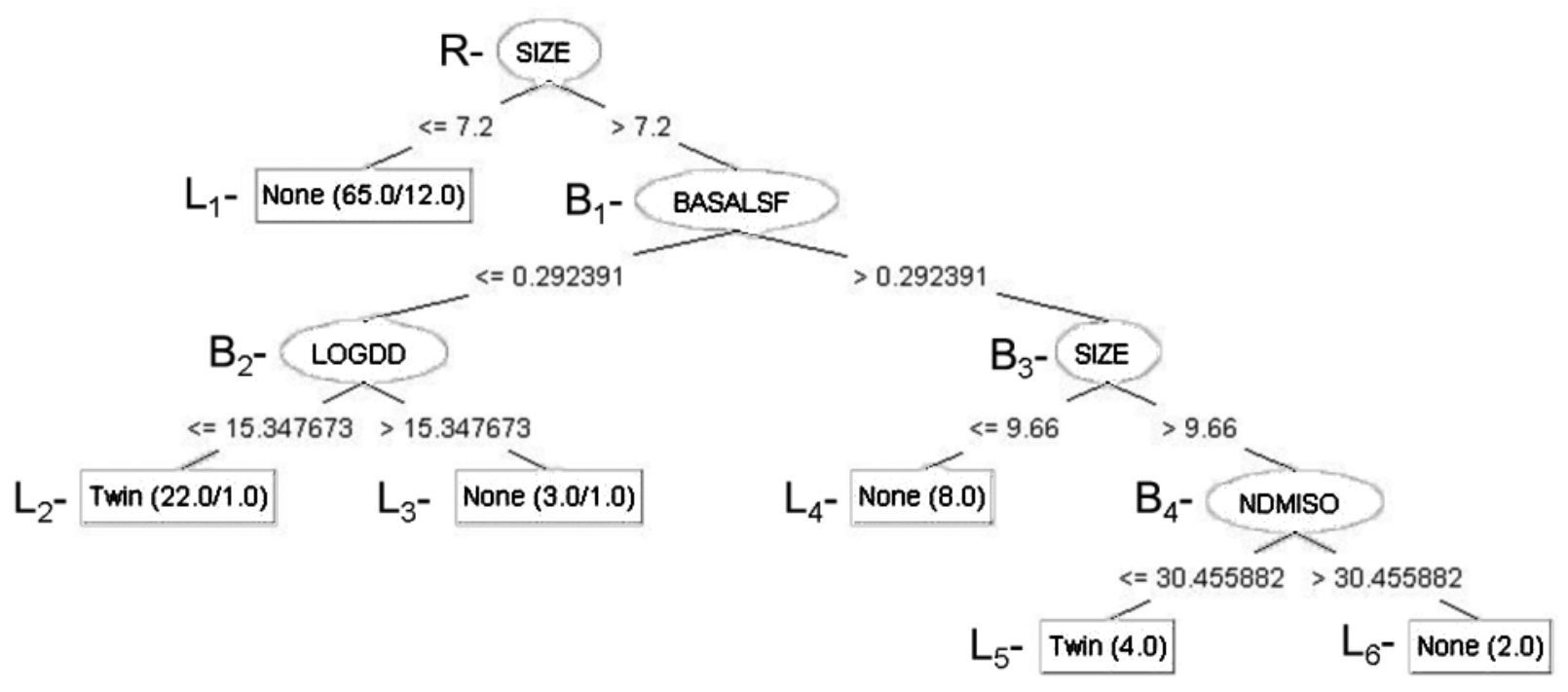

Figure 2: Decision tree for characterizing twin nucleation within an individual grain. Labels $\mathbf{R}, \mathbf{B}$ and $L$ index the root, branches and leaves, respectively. Attribute labels used in the tree are defined in Table 2.

This decision tree is relatively shallow, with only five branches (decision points) and six leaves (end points). As seen in Figure 2, Error! Reference source not found.only 4 attributes were needed to accurately define twin nucleation within a grain. They are, ranked in order of importance:

1. grain size.

2. basal Schmid factor.

3. dislocation density.

4. c-axis to ND deviation.

The presence of grain size in the machine learning model coincides with the findings of many researchers (e.g. [16]). Furthermore, the location of grain size at the "root" of the tree, and a further appearance at branch B3 within the tree, emphasize its fundamental importance. The next attribute of importance in the decision tree is basal Schmid factor; its appearance is in line with the findings of others, as it indicates whether a grain is "hard" or "soft", and therefore prone to twinning (e.g. [41]). The dislocation density and c-axis to ND deviation might be considered new findings pertinent to the twinning phenomenon in $\mathrm{Mg}$. Influence of c-axis misorientation on twinning has previously been proposed for statistical models, but the previous study focused on the somewhat different attribute of misorientations between the c-axis of neighboring grains [66]. These attributes appear near the end of the tree, indicating that they do not provide as much 
information gain as the other attributes, such as grain size. However, if the lower attributes are removed and replaced with random class assignments, the accuracy of the tree on the training data drops by approximately $10 \%$; this suggests that grain size is dominant, but not the exclusive factor in twin formation.

Additionally, the validation of the model is visually ascertained by mapping the predictions made in the decision tree onto the grain structure tested (see

Figure 3: Error maps of decision tree for predicting twinning in individual grains. Microstructure used to build model, 104 grains (left) and test generalization, 1239 grains (right). Correct predictions (blue) and incorrect predictions (red) are shown except for edge grains (gray) which were excluded due to incomplete information.

3). Several insights into the model are made clear by the maps in Figure 3. The first is that the model appears to better predict twin activity in small area grains than large grains, which is likely due to the uneven distribution of instances in the training data. The uneven distribution of training instances can result in a final model that is slightly biased, as seen in the difference in accuracy between small and large grains. It is also seen that misclassified grains are often clustered together, suggesting a limitation in the current model. Twinning is known to often form in localized bands; by using grain based attributes in the model as opposed to local area attributes, this inter-granular interaction is not accounted for. 

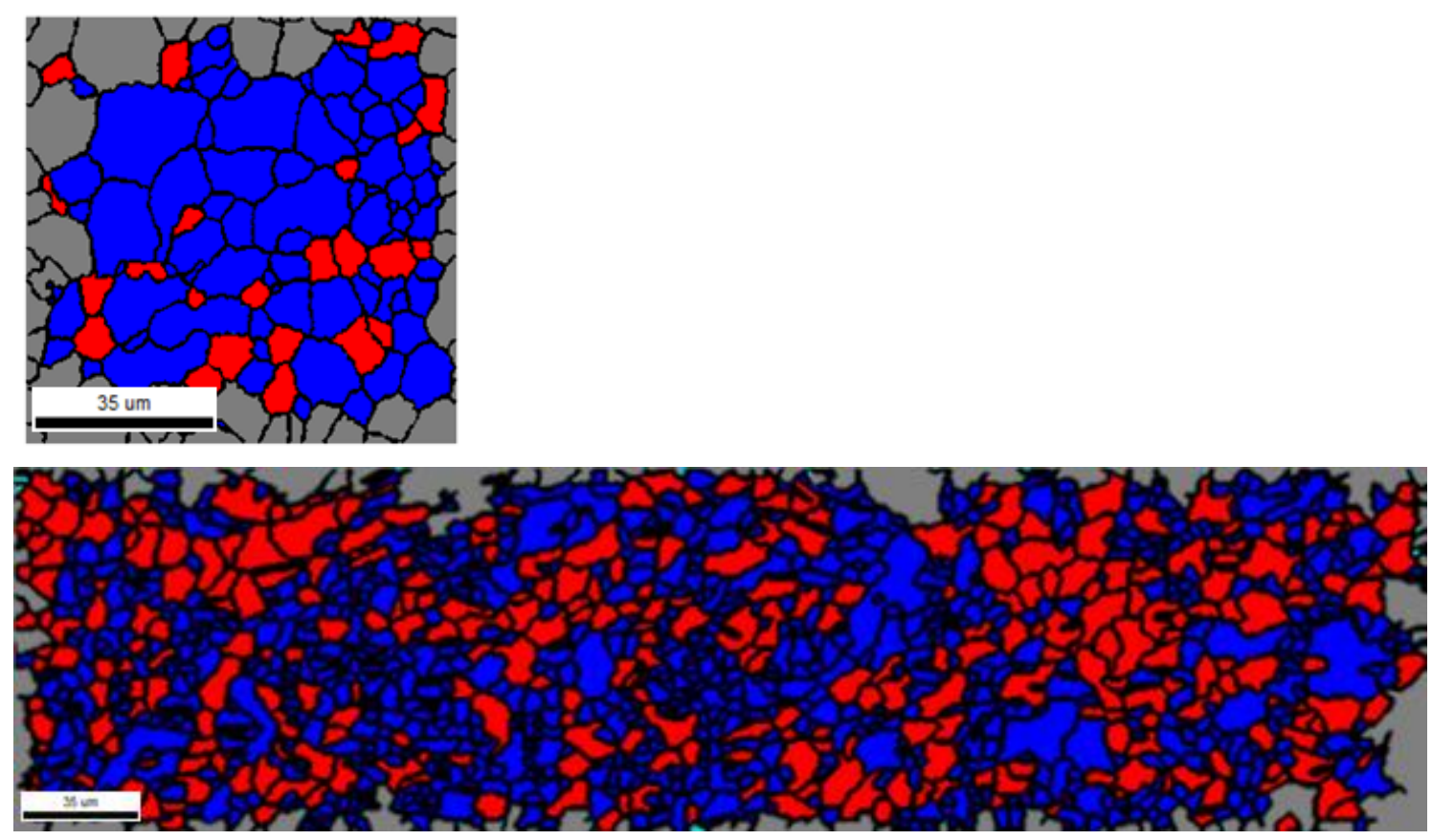

Figure 3: Error maps of decision tree for predicting twinning in individual grains. Microstructure used to build model, 104 grains (left) and test generalization, 1239 grains (right). Correct predictions (blue) and incorrect predictions (red) are shown except for edge grains (gray) which were excluded due to incomplete information.

\subsection{Twin Propagation across Grain Boundaries - Results}


The decision tree model for twin propagation is shown in Figure 4. The model correctly predicted whether a twin propagated across a given grain boundary (GB) in 125 GBs out of the

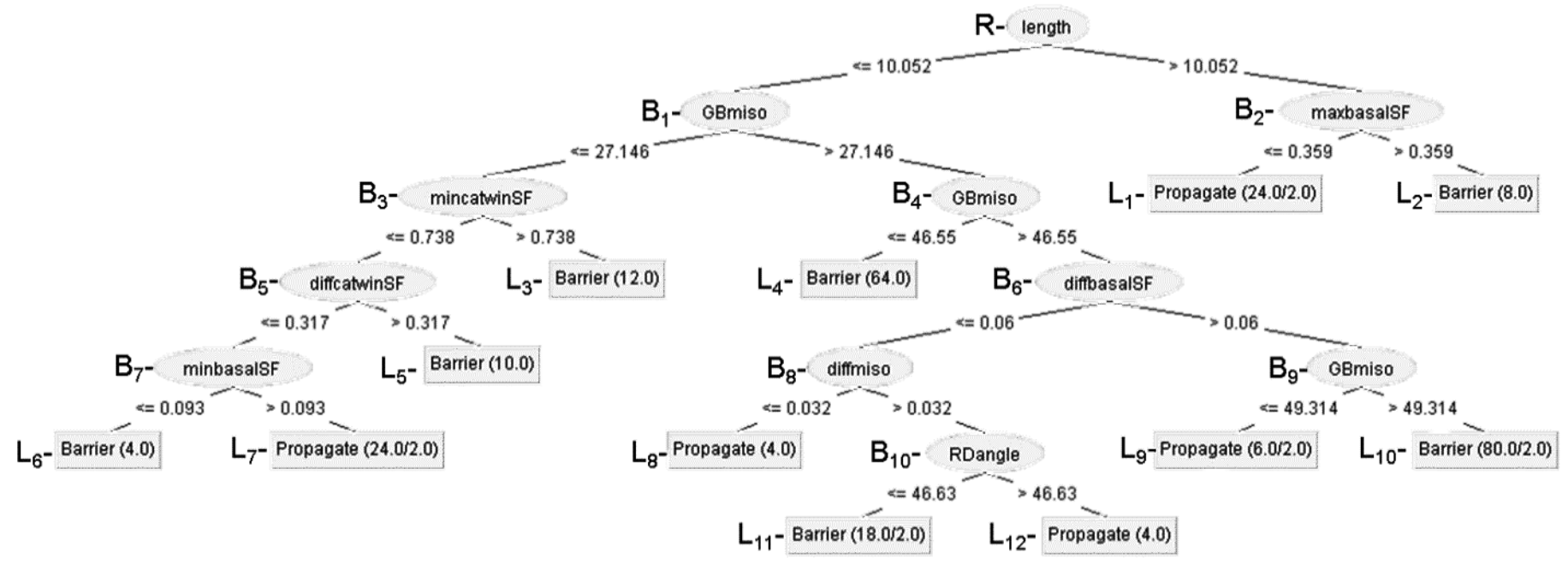

Figure 4: Decision tree for characterizing twin propagation across grain boundaries. Attribute labels used in the tree are defined in Table 2.

130 GBs $(96.1 \%)$ used to create the model, and in 851 GBs out of the 1127 GBs $(75.5 \%)$ used for testing the model. However, the higher accuracy came with an increased depth of decision tree. The nucleation of twins within grains has a relatively small decision tree ( 5 branches and 6 leaves), whereas the model for twin propagation has 11 branches and 12 leaves i.e. approximately twice the size of the prior tree.

In the propagation decision tree, 9 of the possible 19 distinct attributes are present. They are:

1. GB length.

2. GB misorientation.

3. minimum twin to $<\mathrm{c}+\mathrm{a}>\mathrm{Schmid}$ factor ratio.

4. difference of twin to $<c+a>$ Schmid factor across the GB.

5. minimum basal Schmid factor.

6. difference of basal Schmid factor across the GB.

7. difference of kernel average misorientations across the GB.

8. the approximate angle of the GB on the sample surface relative to the RD.

9. the maximum basal Schmid factor. 
Several of the attributes in the model reflect findings from previous work. For example, GB misorientation was considered by both Beyerlein et al [63] and Khosravani et al [41]. The basal Schmid factor appears several times in the tree, confirming the finding by Khosravani et al. that showed the correlation between twin nucleation and basal dislocation buildup at a grain boundary. Other attributes such as GB length capture multiple aspects of prior research such as the grain size effect observed by Barnett [16] and the statistical nature of twinning at a boundary observed by Beyerlein, since larger boundaries have more potential twin nucleation sites.

In order to visualize the accuracy of the GB propagation model, a different type of map is required from that shown in Figure 3. In this case, twin boundaries were colored as either allowing or blocking propagation. Incorrect predictions in either case were highlighted as seen in Figure 5.
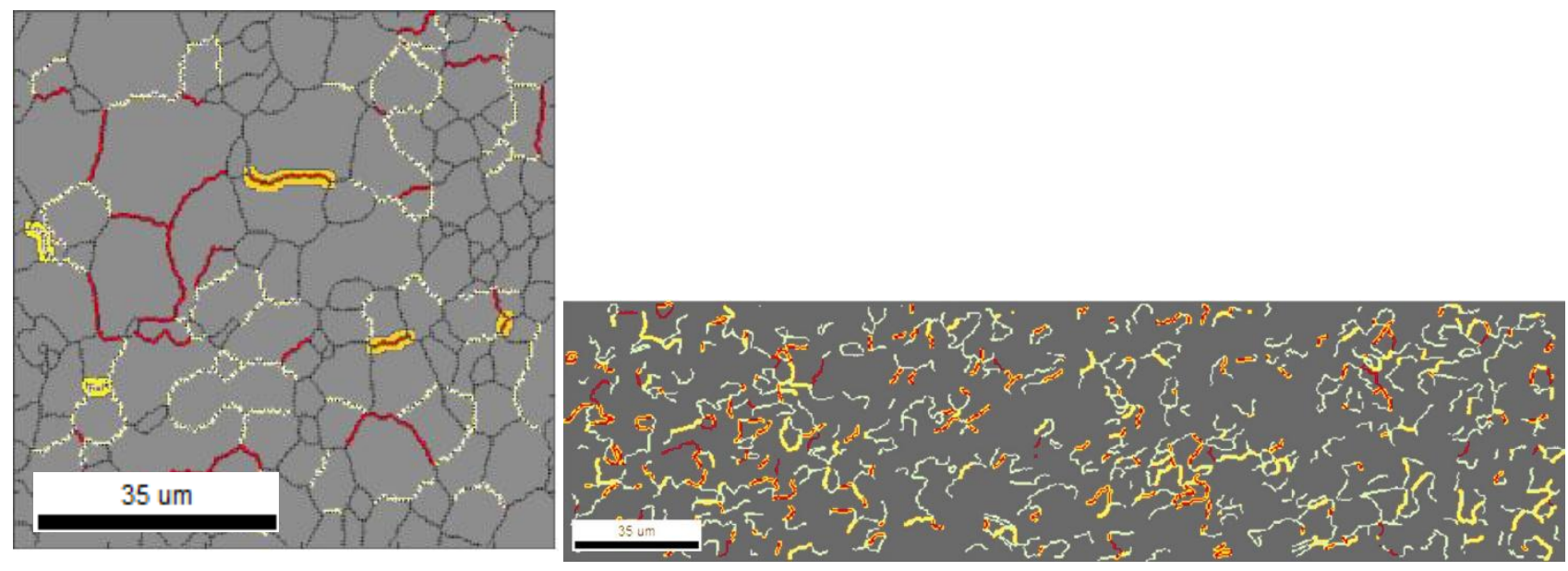

Figure 5: Error maps of the decision tree for predicting twin propagation across grain boundaries. Microstructure used to build model, 130 GBs (left) and test generalization, 1127 GBs (right). GBs that were predicted to allow propagation of twins are shown in red while those that predicted barriers to twin propagation are pale. incorrect prediction assignments are highlighted in yellow (appears orange in the right image). Grain boundaries that do not intersect twins are displayed in black. 


\section{DISCUSSION}

\subsection{Twin Nucleation in Grains - Analysis of Model}

In analyzing the results and accuracy of the decision tree models, the potential benefits include both a twin prediction model (in this case, provided by the decision tree), and detailed insights into microstructure attributes that contribute most to twin activity. An analysis of the grain nucleation model (Figure 2) reveals a hierarchy of attributes that influence twin formation. The detailed correlations between these attributes and twin activity can be explored in more depth using standard statistical analysis. The relative frequency of twinning across the range of values of selected attributes is shown in Figure 6 revealing functional dependence which is not entirely captured in the decision tree, and aids the discussion of the overall performance of the decision tree approach.

The most critical contributor to twin formation within a grain is grain size, at the root $(\mathrm{R})$ of the decision tree. At this point the tree splits into a terminal leaf $\left(\mathrm{L}_{1}\right)$ and a branch $\left(\mathrm{B}_{1}\right)$. This initial division on the tree, dividing grains that are greater or smaller than $7.2 \mu \mathrm{m}$, illustrates the difficulty of twin nucleation in relatively smaller grains, consistent with the Hall-Petch approach on the twinning shear stress described by Barnett [16]. This also aligns with the stochastic view of twinning [67], where smaller grains would be less likely to nucleate twins due to fewer available nucleation sites. A more detailed view of this relationship can be seen in Figure 6c, where increasing grain size generally results in increased likelihood of twinning. However, leaf $\mathrm{L}_{1}$ has a relatively high error (12 of 65 predictions were incorrect), highlighting one negative aspect of the decision tree approach, representing non-linear data using discrete divisions. For example, Figure 6c displays a somewhat non-linear relationship between grain size and twin occurrence; however, the decision tree creates a discrete division based on a single grain size to segregate grains that are more likely to twin from those that are less likely to twin. The nonlinear relationship can be partially incorporated into the tree by the appearance of multiple branches containing the same attribute (as in branch B3 in this tree). More sophisticated machine learning algorithms can be used to better capture the non-linear functional dependence (for example, neural networks); however, such algorithms are not as good at quantifying the relative 
importance of the attributes and thus at clarifying the physics underlying the phenomena of interest.

Another source of error relating to grain size correlations is inherent in the nature of the data being used. EBSD infers a grain size from a 2D slice of the grain. While on average, stereological values of grain size have been shown to be correct [68], there will be various cases when a large grain is assumed to be small due to the fact that it has been sliced near to one end. This type of error will reduce as the size of the data set increases.

The $\mathrm{B}_{1}$ branch in Figure 2 partitions the remaining instances into two sets based upon the basal Schmid factor being greater or less than $\sim 0.29$. Once again, the relevant chart in Figure $6 \mathrm{a}$ displays a more complex relationship between Schmid factor and twin probability; nevertheless, the simple cutoff value approach in the model highlights the fact that lower Schmid values correlate with a higher tendency to twin, potentially owing to the lack of appropriately oriented basal slip systems to accommodate strain.
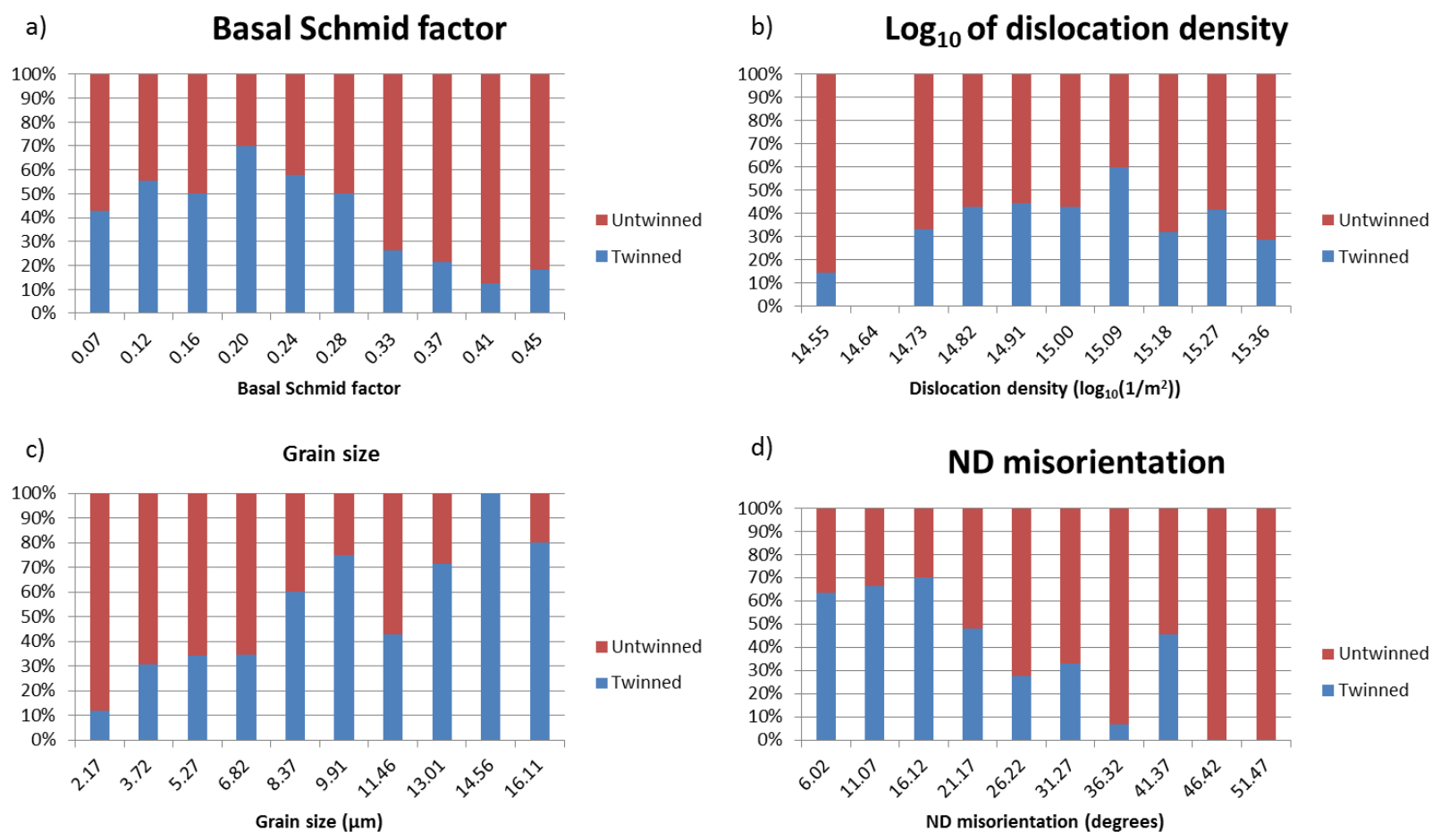

Figure 6: Bar charts of relevant features used in the decision tree for the twin nucleation model: basal Schmid factor (a), dislocation density (b), grain size (c), and ND deviation from the c-axis (d). 
Of lesser influence in the production of twins is dislocation density, as apparent at branch $\mathrm{B}_{2}$, immediately below the basal Schmid factor branch $\left(\mathrm{B}_{1}\right)$, indicating a correlation between lower dislocation content $\left(<2.23 \mathrm{e}^{2} \mathrm{~m}^{-2}\right)$ and twin activity. This is presumably because of the presence of greater slip activity which reduces the necessity for strain accommodation by twinning. Such dependence may not have been immediately apparent from a simple view of the relationship between dislocation density and twin activity, as seen in Figure 6b. The strong correlation comes to light only after the small grains and those with low basal Schmid factors are filtered out using the decision tree approach.

The additional consideration of c-axis to sample-ND deviation contributes the last unique attribute found in the machine learning model, and is found in branch $\mathrm{B}_{4}$. This attribute is used as a measure of how strongly aligned a grain is with the basal texture found in the tested samples (i.e. distinguishing "hard" and "soft" grains). The final leaves $\left(\mathrm{L}_{5}, \mathrm{~L}_{6}\right)$ of the model indicate that grains which are closer to the basal texture tend to twin more, presumably because of the need to accommodate $\mathrm{c}$-axis expansion by means other than <a $>$ type slip. Again, the statistical analysis in Figure 6d provides further insight into the nature of this correlation by illustrating the trend that lower ND misorientations (stronger basal orientation) twin more frequently.

\subsection{Twin Propagation across Grain Boundaries - Analysis of Model}

While the model presented for finding grains containing twins was relatively simple, the decision tree for characterizing propagation of twins across GBs (Figure 4) involved a larger number of parameters and was more complex. This is also highlighted by the statistical correlations of underlying dependencies for various attributes, as shown in Figure 7. Therefore, the focus of the analysis of this model will not be to explain the tree in its entirety but to extract as many reliable conclusions as possible.

The first attribute that appears at the root of the decision tree $(\mathrm{R})$ for propagation is GB length, and attribute that is not always considered in twin models. However, clearly larger GB areas statistically increase the probability of twins nucleating or propagating across a boundary; longer grain boundaries propagate twins more readily than their shorter counterparts $(\leq 10.1 \mu \mathrm{m}$.

The next most important attribute in the decision tree is GB misorientation $\left(\mathrm{B}_{1}\right)$, which had been previously studied by other researchers (e.g. [63]). In the machine learning model, GB misorientation appears at three distinct branches $\left(\mathrm{B}_{1}, \mathrm{~B}_{4}, \mathrm{~B}_{9}\right)$, pointing to a more complex 
relationship between misorientation and twin activity than might previously have been captured by models in the literature. In general, grain boundaries with lower misorientation are more likely to propagate twins while higher misorientation grain boundaries serve as barriers to twin propagation. The machine learning model identified $27.1^{\circ}$ as the approximate threshold angle of misorientation below which propagation is favored, showing good agreement with the $25-39^{\circ}$ found by Khosravani et al [41]. Note that this apparent correlation may also be due to the fact that hard grains neighboring soft grains tend to have high angle boundaries, and that, strain accommodated in the soft grain by slip must be accommodated in the hard grain by twins (see the discussion in [41]). However, the overall effect of GB misorientation on twin propagation is fairly complex, as seen in Figure 7b. This complexity is expressed in the decision tree model by the reoccurrence of GB misorientation. It is interesting to note the presence of spikes in twin propagation instances in Figure 7b. While not investigated in this work, these could correlate with special grain boundary types.
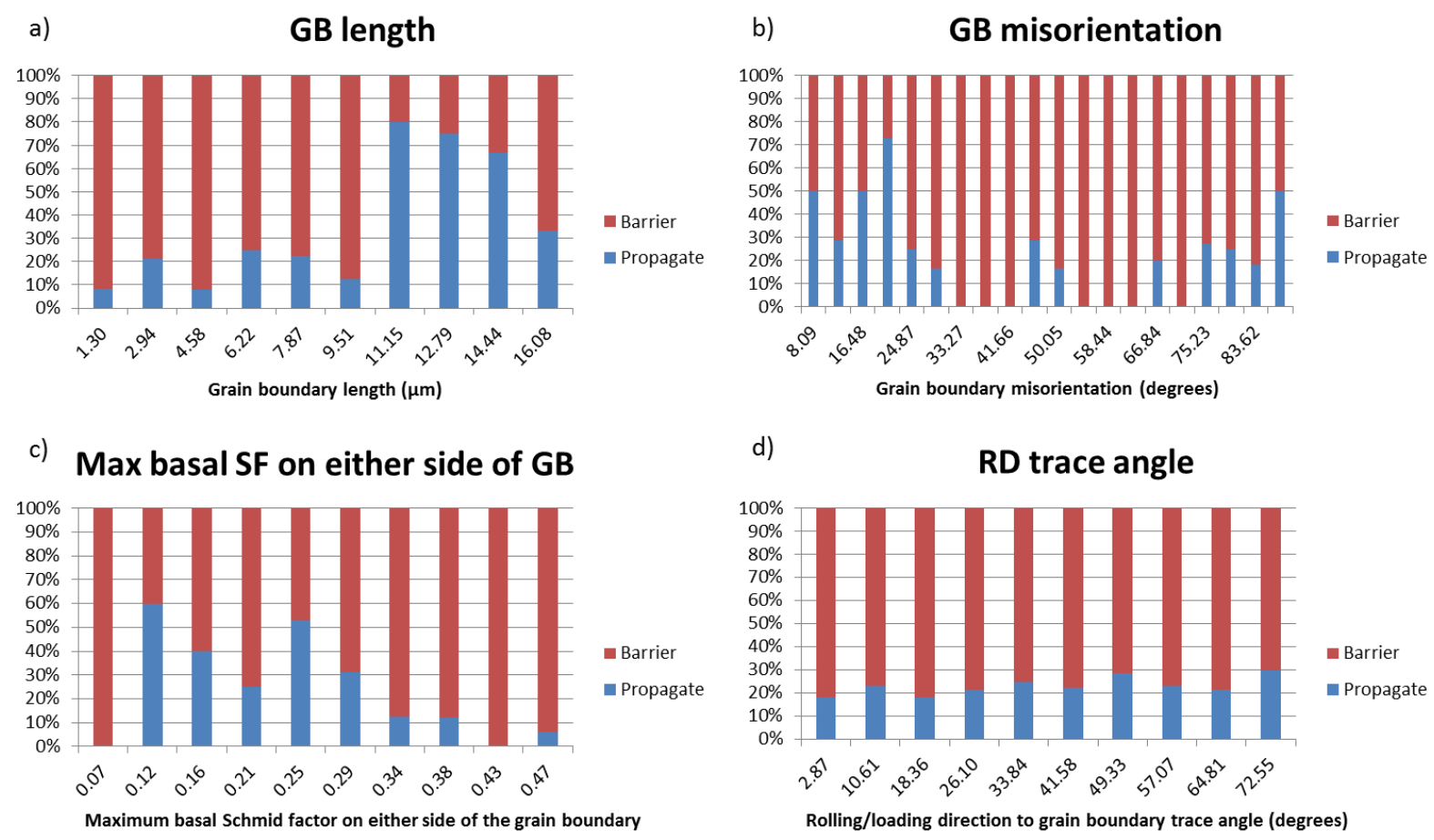

Figure 7: Bar charts of relevant features used in the decision tree for the twin propagation model: grain boundary length (a), grain boundary misorientation (b), maximum basal Schmid factor (c), and the angle between the grain boundary trace and loading direction, $\mathrm{RD}(\mathrm{d})$. 
Another significant attribute in the machine learning model is the basal Schmid factor near grain boundaries $\left(\mathrm{B}_{2}, \mathrm{~B}_{6}, \mathrm{~B}_{7}\right)$. As presented in the decision tree, the maximum basal Schmid factor is pivotal in deciding whether longer grain boundaries block or propagate twins. This can also be visualized to some extent via the basal SF plot in Figure 7c. According to $\mathrm{B}_{2}$, when the maximum basal SF on either side of a boundary is less than $\sim 0.36$, twins tend to propagate. If basal slip systems cannot easily accommodate the stress state caused by a twin on the opposing side of a GB then twinning may occur in order to accommodate that stress. Previous research has demonstrated the stress rise at GBs and triple junctions, using finite element modeling, for various materials and triple junction configurations $[69,70]$. In these studies, the stress at a triple junction has been shown to be as much as $30 \%$ higher than the average stress in the grain. Referring back to Error! Reference source not found. this increase in stress may accommodate the transition from activation of prismatic slip (8-10 MPa) to activation of tensile twinning (11-12 $\mathrm{MPa}$ ). Furthermore, Koike [14] discussed the formation of twins due to stress concentration and Barnett [11] showed that twins intersecting other boundaries can lead to void/crack nucleation which also serves to illustrate the increased stresses at these locations. In brief, the stress concentration caused by a twin intersecting a GB may be sufficient to increase the local stress above the threshold for tensile twinning, causing that twin to propagate across the boundary.

Other attributes present in the decision tree include the ratio of twin Schmid factor to $<\mathrm{c}+\mathrm{a}>$ Schmid factor $\left(\mathrm{B}_{3}, \mathrm{~B}_{5}\right)$, kernel average misorientation $\left(\mathrm{B}_{8}\right)$, and the average angle between the GB and loading direction $\left(\mathrm{B}_{10}\right)$. The ratio of Schmid factors $\left(\mathrm{B}_{3}, \mathrm{~B}_{5}\right)$ provides information as to whether incompatibility will exist at a GB which would then need to be accommodated by a twin. Smaller differences between these ratios across a GB may encourage twins to propagate, since both grains are more likely to twin than activate $<c+a>$ slip. Additionally, kernel average misorientation $\left(\mathrm{B}_{8}\right)$ has been used by some researchers as an estimate of dislocation activity [71]. In the decision tree smaller differences across a GB tend to result in propagation of twins, and larger differences serve as barriers. Similar observations were made by Khosravani that suggested that dislocation pileups can correlate with poor twin propagation as well as spontaneous twin nucleation [41]. The final morphological measure of grain boundary trace angle $\left(\mathrm{B}_{10}\right)$ seems to show that boundaries more perpendicular to the RD will propagate twins, likely due to the increased area across which the incoherent twin boundary spans. This 
relationship cannot be seen clearly in Figure $7 \mathrm{~d}$ but is extracted through the machine learning algorithm pointing to a potential advantage of the method presented in this paper.

The decision tree is very accurate for the original data set from which it was derived, but less so for the larger test data. This may be due, in part, to the large size of the tree. Large trees / models tend to increase the likelihood of over fitting which limits the models ability to accurately characterize generalized data sets. Regardless of the potential errors in this model, attributes nearest to the root of the tree would most likely remain the most important across more general datasets, thus maintaining the relative accuracy and interpretation of the model.

\subsection{Machine Learning Framework - Discussion}

When selecting the decision tree algorithm, it was understood that it would provide a rule-based description of the studied events related to twinning in AZ31, and not a knowledgebased description. Knowledge based machine learning methods, such as neural networks, tend to produce more accurate models, but require prior knowledge about the event studied. The methods employed by using J48 do not require previous knowledge about the events and thus cannot create the more complex, yet more accurate, knowledge based results. While trade off of accuracy might be considered a weakness of this machine learning framework, it is in fact an advantage since understanding of twin nucleation and propagation is not sufficient to create a model based on prior knowledge. The idea of extracting structure/ phenomena relations and ranking the importance of different structure attributes, without prior understanding of the physics, is perhaps the greatest benefit of using this rule-based machine learning approach. For example, if one considers a scenario where no previous work had been done to study twinning in $\mathrm{Mg}$, then the results of machine learning could have focused subsequent studies on the highlighted individual attributes found in the decision trees. Even as prior knowledge was considered here, modest insights beyond current models have been identified for deformation twinning in AZ31.

The difference in accuracy between the small and large dataset should be noted. The models did not perform as well on the larger datasets, likely for several reasons. First, the model is biased towards the small dataset, as it was used to train the model. Second, small differences in strain or texture between the samples could greatly affect their accuracy. Despite the differences in accuracy, the $75 \%$ accuracy of the model in comparison to the large dataset 
confirms that the framework produces robust models that have potential to be deployed for use with new data.

For example, one significant attribute found using the machine learning framework is the deviation of the c-axis from the sample ND. This new knowledge could lead to a more in-depth study to observe highly non-basal grains in a mostly basal textured material in an effort to come up with a measure of strain incompatibility that would then help explain twinning in these grains.

Another potential application of machine learning as it applies to the findings of this paper is the opportunity to refine and accelerate microscope-based studies on twinning in $\mathrm{Mg}$ AZ31. The knowledge gained via the machine learning method gives future researchers the ability to quickly select areas of interest (e.g. regions where twin nucleation is probable) and capture high quality data in these areas. The model may also be applied to observe twin nucleation at its earliest detectable stages by looking for the highlighted attributes at lower levels of global strain. Such tools could serve to help future studies of any complex materials phenomenon observable by EBSD.

\section{CONCLUSION}

Twin formation within grains and twin propagation across grain boundaries in $\mathrm{Mg}$ alloy AZ31 was studied using a machine learning framework (J48 decision tree), which extracted attribute based rules. Models emerging from the study were $86.5 \%$ accurate for predicting twin nucleation and $96.1 \%$ accurate for predicting twin propagation across grain boundaries against the cross validated training set of 104 grains. The accuracy of the models against an independent test set was $75.1 \%$ for twin nucleation in 1239 grains and the twin propagation model was $75.5 \%$ accurate on 1127 GBs. The compression strain and distribution of twinned vs untwinned instances were slightly different between the test and model samples, which may have led to discrepancies between the accuracies between the two datasets. 
The important observations associated with twin nucleation on the grain level, in order of the greatest to lowest relevance, include:

- increased twin nucleation in larger grains $(>7.2 \mu \mathrm{m})$

- decreased twin nucleation in grains with maximum basal Schmid factors above $\sim 0.29$

- decreased twin nucleation in grains with average dislocation densities above $2.23 \mathrm{e} 15 \mathrm{~m}^{-2}$

- increased twin nucleation in grains where the angular deviation between c-axis and sample ND was less than $30.5^{\circ}$

The important observations associated with twin propagation across a grain boundary, in order of relevance include:

- increased twin propagation across GBs with a length greater than $10.05 \mu \mathrm{m}$

- increased twin propagation at GB misorientations $<27.1^{\circ}$

- decreased twin propagation for GBs where the maximum basal Schmid factor exceeded 0.359

- increased twin propagation across GBs where the difference between the ratio of twin Schmid factor to $<\mathrm{c}+\mathrm{a}>\mathrm{Schmid}$ factor was less than or equal to 0.317

- decreased twin propagation across GBs with a difference between kernel average misorientation greater than $0.032^{\circ}$

- decreased twin propagation across GBs where the average angle between the GB and loading direction was less than or equal to $46.63^{\circ}$

The combination of these attributes provide the greatest overall information gain of the available features and help understand the physical processes associated with tensile twinning in AZ31. Furthermore, the findings of this paper stand in agreement with work done by previous researchers (grain size corresponds to Hall-Petch/Barnett models [16], boundary length corresponds to Beyerlein [17], and GB misorientation corresponds to both Khosravani [41] and Beyerlein [17]) while building upon it with new insights. These additional insights provide a deeper understanding of some of the previously hidden details affecting twinning in AZ31, leading to potentially more accurate models in future work.

In conjunction with the findings on twinning in this $\mathrm{Mg}$ alloy, a machine learning framework for utilizing EBSD data has been adopted that can be used to analyze a variety of complex phenomena. The benefits of this technique are two-fold. They are; i) underlying processes, or 
microstructural features, behind studied events can be quickly revealed, and ii) continued study of a phenomenon will be aided by the ability to quickly refine data collection to areas of interest in a microstructure. The framework presented in this study is not limited to twinning in $\mathrm{Mg}$ or its alloys but extends to the broader set of crystalline materials. Ultimately this technique has potential for analyzing complex metallographic phenomena rapidly, which will allow for acceleration in researching links between microstructure and material properties.

\section{Acknowledgements}

Research was supported by U.S. Department of Energy (DOE), Office of Science, Basic Energy Sciences (BES), under Award \#DE-SC0012587 (computational studies and theory development), and by the National Science Foundation (NSF), under award \#CMMI 1404771 (experimental data gathering activities). 


\section{REFERENCES}

[1] B.L. Mordike, T. Ebert, Materials Science and Engineering: A, 302 (2001) 37-45.

[2] T. Al-Samman, G. Gottstein, Materials Science and Engineering A, 488 (2008) 406-414.

[3] W.A.B. B.C. Wonziewiz Transactions of the Metallurgical Society of AIME, 239 (1967) $1422-1433$.

[4] A. Chapuis, J. Driver, Journal of Physics: Conference Series, 240 (2010) 012092.

[5] A. Chapuis, J.H. Driver, Acta Mater, 59 (2011) 1986-1994.

[6] P. Klimanek, A. Pötzsch, Materials Science and Engineering: A, 324 (2002) 145-150.

[7] R.N. N Ono, S Miura, Materials Letters, 58 (2003) 39-43.

[8] K. Piao, K. Chung, M.G. Lee, R.H. Wagoner, Metall Mater Trans A, 43A (2012) 3300-3313.

[9] L.X. Wagoner RH, Li M, Agnew SR, , .; J. Mater. Proc. Tech, (2006) 483-485.

[10] P. Yang, L. Meng, Y. Hu, Z. Zhao, X. Ren, Image Anal Stereol, 23 (2004) 53-61.

[11] M.R. Barnett, Mat Sci Eng a-Struct, 464 (2007) 8-16.

[12] M.R. Barnett, Materials Science and Engineering: A, 464 (2007) 1-7.

[13] R.H. J Wang, J Hirth, L Capolungo, I Beyerlein, C Tome, Scripta materialia, 61 (2009) 903906.

[14] J. Koike, Metall. Mater. Trans.A, 36 (2005) 1689-1696.

[15] L. Meng, P. Yang, Q. Xie, W. Mao, Materials Transactions, 49 (2008) 710-714.

[16] M.R. Barnett, Z. Keshavarz, A.G. Beer, D. Atwell, Acta Mater, 52 (2004) 5093-5103.

[17] I. Beyerlein, R. McCabe, C. Tomé, Journal of the Mechanics and Physics of Solids, (2011).

[18] L. Capolungo, I.J. Beyerlein, C.N. Tome, Scripta materialia, 60 (2009) 32-35.

[19] I.B. J Wang, C Tome, Scripta materialia, 63 (2010) 741-746.

[20] J.J. Jonas, S. Mu, T. Al-Samman, G. Gottstein, L. Jiang, Ė. Martin, Acta Mater, 59 (2011) 2046-2056.

[21] M.A. Meyers, O. Vohringer, V.A. Lubarda, Acta Mater, 49 (2001) 4025-4039.

[22] B.L. Adams, Ultramicroscopy, 67 (1997) 11-17.

[23] B.L. Adams, S.I. Wright, K. Kunze, Metallurgical and Materials Transactions A, 24 (1993) 819-831.

[24] T.M. Maitland, A. Gholinia, Microsc Microanal, 13 (2007) 924-925.

[25] J. Basinger, D. Fullwood, B. Adams, TMS, San Diego, 2011.

[26] C.J. Gardner, B.L. Adams, J. Basinger, D.T. Fullwood, International Journal of Plasticity, 26 (2010) 1234-1247.

[27] S. Villert, C. Maurice, C. Wyon, R. Fortunier, Journal of Microscopy, 233 (2009) 290-301.

[28] A.J. Wilkinson, G. Meaden, D.J. Dingley, Microsc Microanal, 11 (2005) 520-521.

[29] Y. Reich, N. Travitzky, Materials \& design, 16 (1995) 251-259.

[30] W. Sha, K. Edwards, Materials \& design, 28 (2007) 1747-1752.

[31] A. Tompos, J.L. Margitfalvi, E. Tfirst, K. Héberger, Applied Catalysis A: General, 324 (2007) 90-93.

[32] R. Yoram, T. Nahum.

[33] S. Agnew, O. Duygulu, Int. J. Plasticity, 21 (2005) 1161-1193.

[34] S. Graff, W. Brocks, D. Steglich, International Journal of Plasticity, 23 (2007) 2007.

[35] A. Izadbakhsh, K. Inal, R.K. Mishra, M. Niewczas, Comp Mater Sci, 50 (2011) 2185-2202. 
[36] J.W. Christian, S. Mahajan, Prog Mater Sci, 39 (1995) 1-157.

[37] G.I. Taylor, Journal of the Institute of Metals, 62 (1938) 307-324.

[38] S.R. Agnew, C.N. Tome, D.W. Brown, T.M. Holden, S.C. Vogel, Scripta materialia, 48 (2003) 1003-1008.

[39] S.R. Agnew, M.H. Yoo, C.N. Tomé, Acta Mater, 49 (2001) 4277-4289.

[40] J. Koike, T. Kobayashi, T. Mukai, H. Watanabe, M. Suzuki, K. Maruyama, K. Higashi, Acta Mater, 51 (2003) 2055-2065.

[41] A. Khosravani, D. Fullwood, J. Scott, M. Miles, R. Mishra, Acta Mater, 100 (2015) 202214.

[42] S. Wright, M. Nowell, J. Basinger, Microscopy \& Microanalysis, 17 (2011) 406-407.

[43] EDAX-TSL, 2010.

[44] T.B. Britton, A.J. Wilkinson, Ultramicroscopy, 111 (2011) 1395-1404.

[45] J. Kacher, B. Adams, D. Fullwood, MS\&T, Pittsburgh, 2009.

[46] C. Landon, B. Adams, J. Kacher, Journal of Engineering Materials and Technology, 130 (2008) 40-45.

[47] A.J. Wilkinson, G. Meaden, D.J. Dingley, Materials Science and Technology, 22 (2006) 111.

[48] N. Altinkok, R. Koker, Materials \& design, 27 (2006) 625-631.

[49] R. Haj-Ali, H.K. Kim, S.W. Koh, A. Saxena, R. Tummala, International Journal of Plasticity, 24 (2008) 371-396.

[50] R. Koker, N. Altinkok, A. Demir, Materials \& design, 28 (2007) 616-627.

[51] J. Pérez-Benitez, L. Padovese, Expert Systems with Applications, (2011).

[52] N. Reddy, A. Rao, M. Chakraborty, B. Murty, Materials Science and Engineering: A, 391 (2005) 131-140.

[53] L. Xu, L. Wencong, J. Shengli, L. Yawei, C. Nianyi, Chemometrics and intelligent laboratory systems, 82 (2006) 8-14.

[54] R.S. Yassar, O. AbuOmar, E. Hansen, M.F. Horstemeyer, Materials \& design, 31 (2010) 3683-3689.

[55] J. Rasmussen, IEEE Transaction on Systems, Man, and Cybernetics, 13 (1983) 257-266.

[56] J.R. Quinlan, J Artif Intell Res, 4 (1996) 77-90.

[57] M.M. Jon Scott, David Fullwood, Brent Adams, Ali Khosravani, Raja K. Mishra, Metall. Mater. Trans.A, 44 (2012) 512-516.

[58] I. EDAX, EDAX-TSL, 2010.

[59] BYU, github.com, 2015.

[60] I. The Mathworks, The Mathworks, Inc., 2006.

[61] The Mathworks, Inc., 2006.

[62] I.J. Beyerlein, C.N. Tome, Proceedings of the Royal Society A, 466 (2010) 2517-2544.

[63] I.J. Beyerlein, R.J.McCabe, C.N.Tome, Journal of the Mechanics and Physics of Solids, 59 (2011) 988-1003.

[64] WEKA, The University of Waikato, Hamilton, New Zealand, 2010.

[65] J.R. Quinlan, Morgan Kaufmann Publishers, (1993).

[66] I.J. Beyerlein, L. Capolungo, P.E. Marshall, R.J. McCabe, C.N. Tomé, Philosophical

Magazine, 90 (2010) 2161-2190.

[67] S.R. Niezgoda, A.K. Kanjarla, I.J. Beyerlein, C.N. Tomé, Int J Plasticity, 56 (2014) 119138.

[68] S.I. Wright, Prakt Metallogr-Pr M, 47 (2010) 16-33. 
[69] J.W.a.Z. Yue, J. Mater. Sci. Technol., 18 (2003) 303-308.

[70] A. Fallahi, A. Ataee, Materials Science and Engineering: A, 527 (2010) 4576-4581.

[71] D.P. Marion Calcagnatto, Eralp Demir, Dierk Raabe, Materials Science and Engineering: A, 527 (2010) 2738-2746. 


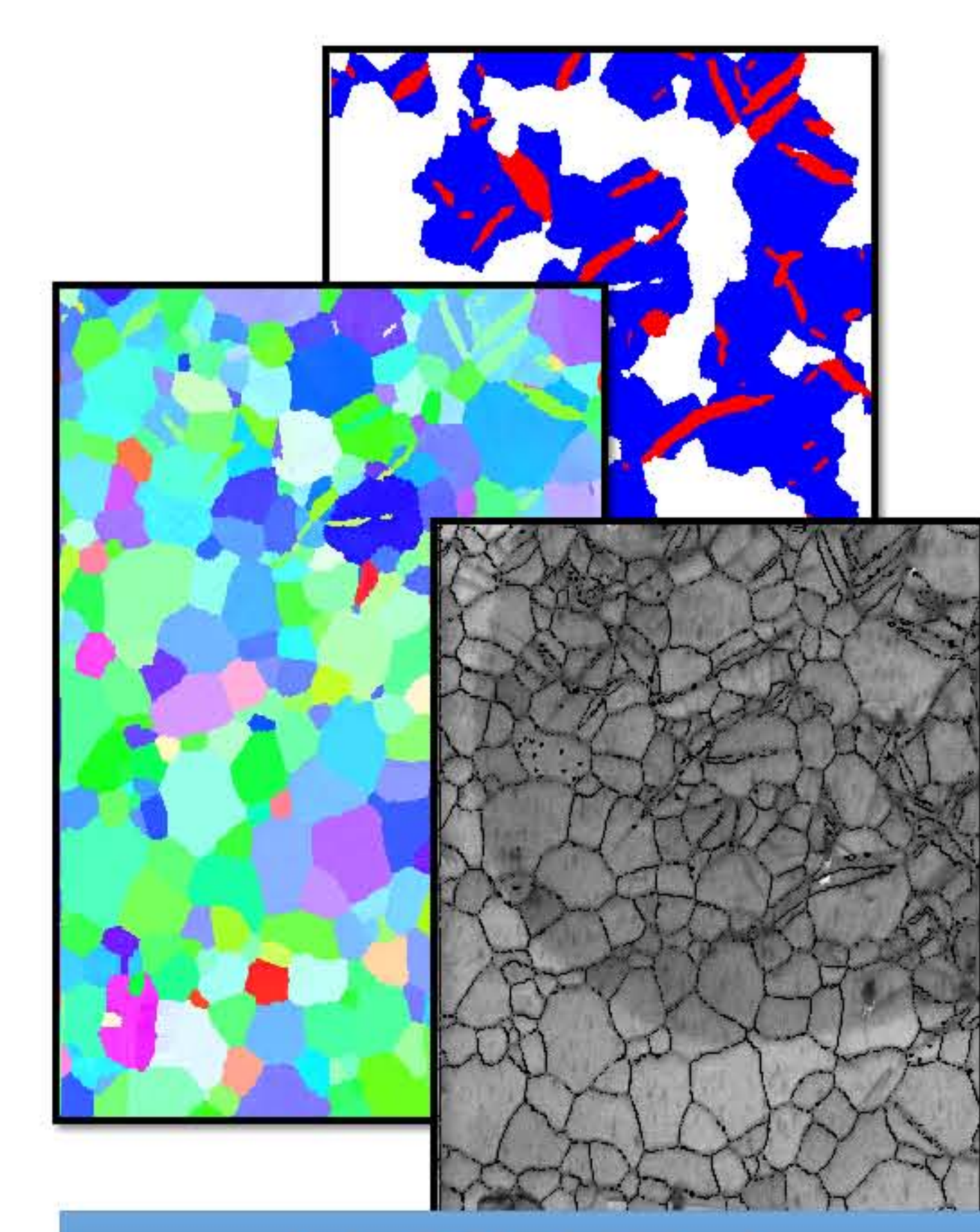

Machine Learning Algorithms

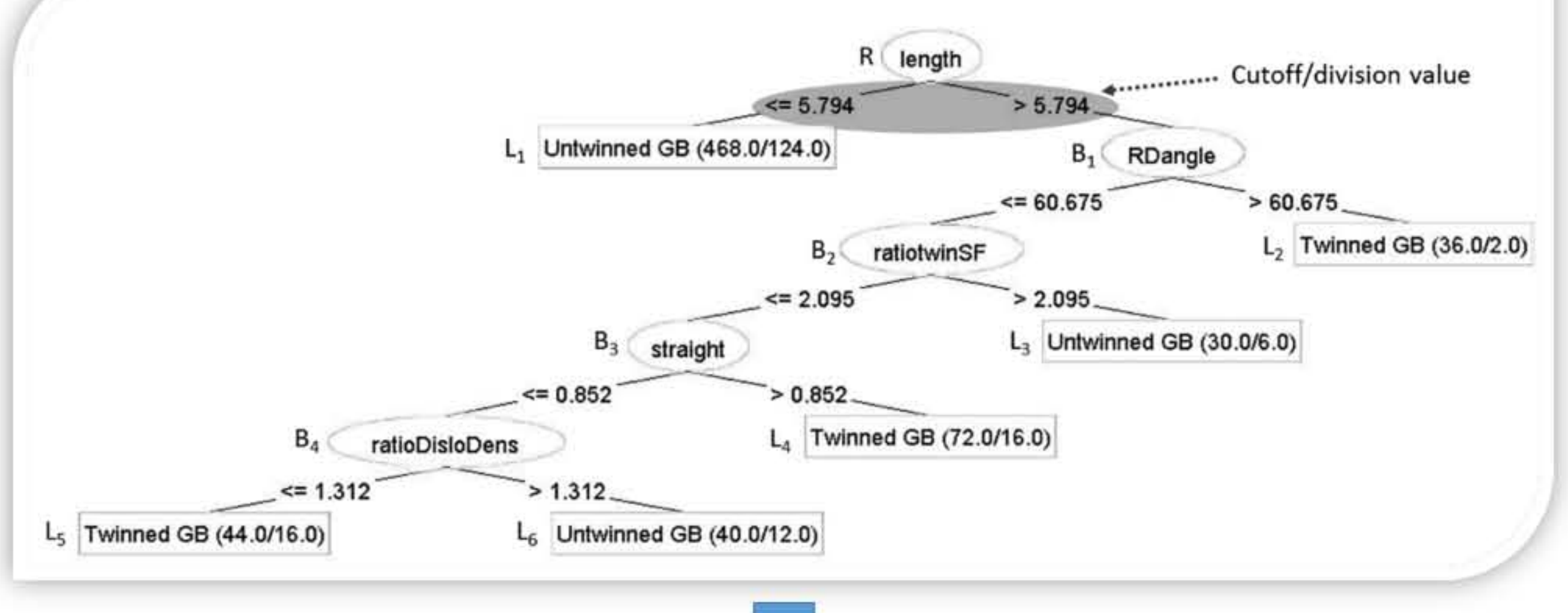

